\title{
Targeting neoplasia using volumetric laser endomicroscopy with laser marking
}

Volumetric laser endomicroscopy (VLE) is a recent advanced imaging technology that allows high resolution microstructure imaging of the esophagus and gastric cardia; it has been increasingly used in Europe and the USA [1 - 3]. The system has been recently upgraded to include a laser marking device that places cautery marks on the mucosa to provide targets for histology. We report a case of an incidental finding of focal high grade dysplasia targeted using this new technology. A 69-year-old man was referred for advanced imaging and removal of a gastroesophageal junction polyp. The polyp was visualized, using high definition resolution white-light endoscopy (HDR-WLE), on the cardia side of the gastroesophageal junction ( $\triangleright$ Fig. 1; Video 1 ). It was a mobile pedunculated polyp with its base at the gastric cardia (Paris classification $0-1 \mathrm{p})$.

It is our practice to use VLE for high resolution imaging of mucosal pathology in the esophagus and gastroesophageal junction. We used a 20-mm balloon containing the VLE probe ( $\mathbf{F i g} \mathbf{2}$ ). VLE showed that the polyp of interest did not contain any of the VLE characteristics that have been associated with neoplasia $[4,5]$. No atypical glands or abnormal signal intensity were seen. There was however an area at the gastroesophageal junction just proximal to the polyp that contained a cluster of atypical glands that were suspicious for neoplasia ( $\triangleright$ Fig.3). This area appeared normal on HDR-WLE and narrow-band imaging (NBI) so laser marks were placed at the site to mark it for targeting ( Fig.4). Endoscopic mucosal resection of the polyp and the VLE-targeted area was performed. Histology of the polyp showed an inflammatory polyp and the VLE-targeted area was consistent with focal high grade dysplasia (> Fig.5; - Video 1).

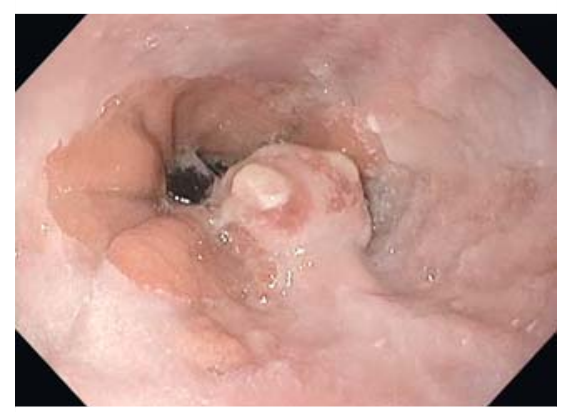

- Fig. 1 Endoscopic view using high definition resolution white-light endoscopy showing the polyp at the gastroesophageal junction and adjacent normal-looking areas.

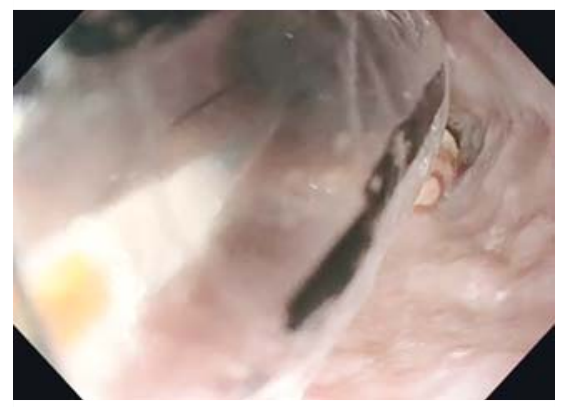

- Fig. 2 The balloon containing the volumetric laser endomicroscopy (VLE) probe scanning the gastroesophageal junction and distal esophagus.
This case demonstrates the capability of VLE to obtain high resolution microstructure imaging of the esophagus and gastroesophageal junction that can aid in the diagnosis of neoplasia.

Endoscopy_UCTN_Code_CCL_1AB_2AC_3AC

Competing interests

None

The Authors

Arvind J. Trindade, Sumant Inamdar, Divyesh V. Sejpal, Arvind Rishi, Keith Sultan Division of Gastroenterology, Hofstra Northwell School of Medicine, Long Island Jewish Medical Center, Northwell Health System, New Hyde Park, New York, USA

\section{Corresponding author}

Arvind J. Trindade, MD

Long Island Jewish Medical Center, Division of Gastroenterology, Hepatology and Nutrition, 270-05 76th Avenue, New Hyde Park, NY 11040, USA

Fax: +1-718-470-5509 arvind.trindade@gmail.com

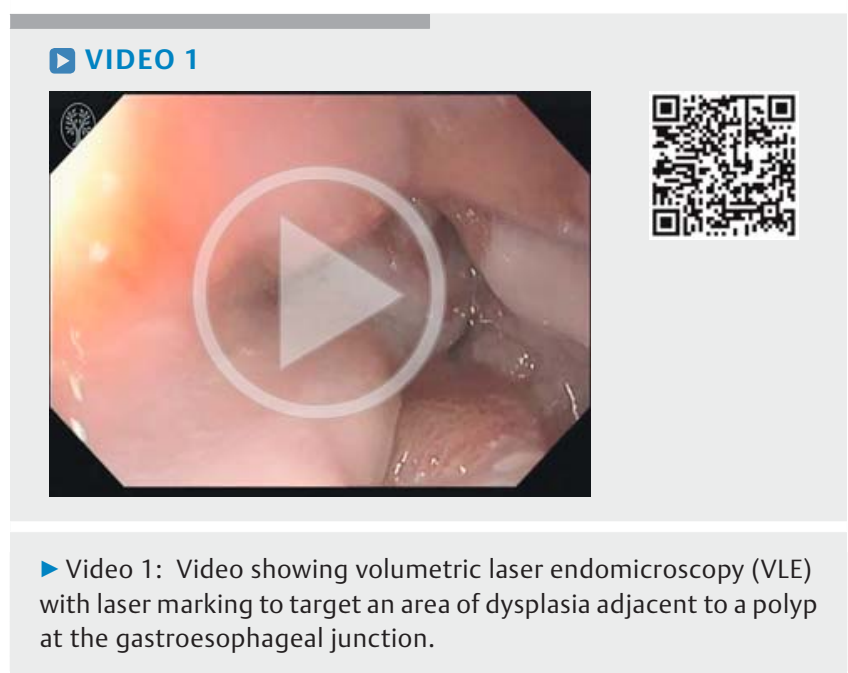




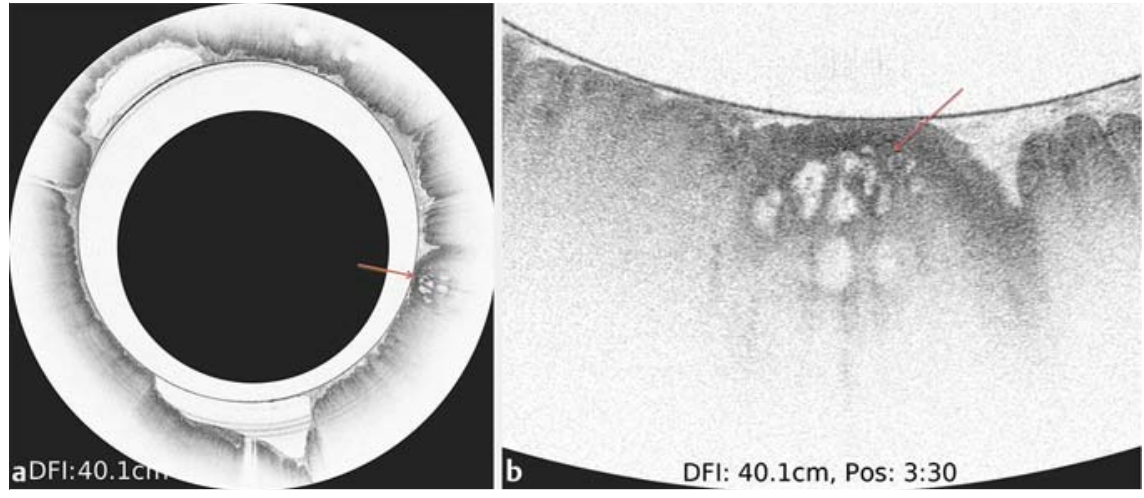

- Fig. 3 Volumetric laser endomicroscopy (VLE) of the area proximal to the polyp showing: a on 360-degree image, an area that was suspicious for neoplasia (arrow); b a magnified snapshot of the suspicious area with an atypical cluster of glands (arrow).

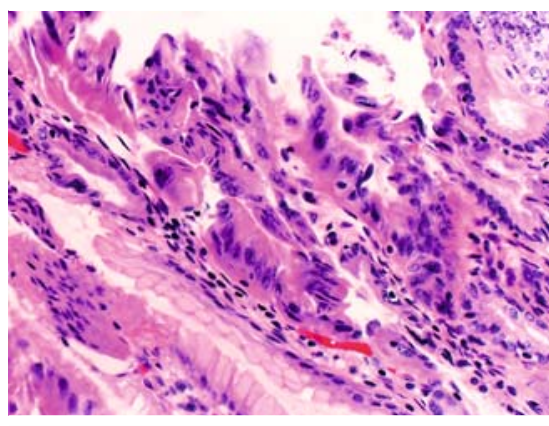

- Fig. 5 Histology of the endoscopic mucosal resection specimen of the volumetric laser endomicroscopy (VLE)-targeted area. Columnar epithelium with loss of surface maturation, architecture complexity and high-grade nuclear atypia (HE $\times 400$ magnification). kid on the block for advanced imaging in

\section{References}

[1] Trindade AJ, Smith MS, Pleskow DK. The new Barrett's esophagus: a review of volumetric laser endomicroscopy. Therap Adv Gastroenterol 2016; 9: 408-416

[2] Wolfsen HC, Sharma P, Wallace MB et al. Safety and feasibility of volumetric laser endomicroscopy in patients with Barrett's esophagus (with videos). Gastrointest Endosc 2015; 82: 631-640

[3] Swager A, Tearney G], Leggett CL et al. Identification of volumetric laser endomicroscopy features predictive for director of endoscopy. Gastrointest Endosc. DOI: 10.1016/j.gie.2016.09.012

[4] Trindade AJ, George B], Berkowitz J et al. Volumetric laser endomicroscopy can target neoplasia not detected by conventional endoscopic measures in long segment Barrett's esophagus. Endosc Int Open 2016; 4: E318-E322

[5] Leggett CL, Gorospe EC, Chan DK et al. Comparative diagnostic performance of volumetric laser endomicroscopy and confocal laser endomicroscopy in the detection of dysplasia associated with Barrett's esophagus. Gastrointest Endosc 2016; 83: 880 888.e2

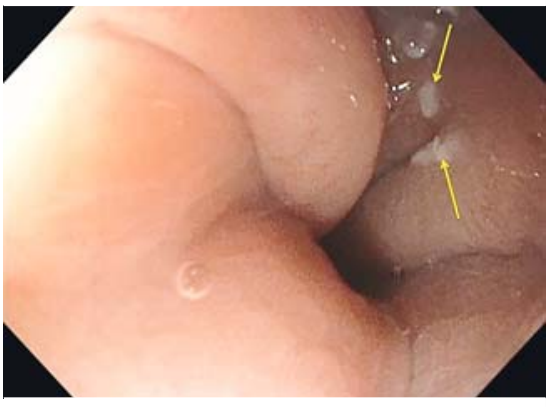

- Fig. 4 Endoscopic view showing laser marks placed at the area of suspected neoplasia using the volumetric laser endomicroscopy (VLE) laser marking system to help target the area for resection (arrows).

\section{Bibliography}

DOI http://dx.doi.org/10.1055/s-0042-122144 Endoscopy 2017; 49: E54-E55

(c) Georg Thieme Verlag KG

Stuttgart · New York

ISSN 0013-726X 